\title{
Role of the mammalian ATG8/LC3 family in autophagy: differential and compensatory roles in the spatiotemporal regulation of autophagy
}

\author{
You-Kyung Lee E Jin-A Lee \\ Department of Biological Sciences and Biotechnology, College of Life Sciences and Nanotechnology, Hannam University, Daejeon 34054, \\ Korea
}

\begin{abstract}
Autophagy, an evolutionarily conserved cellular degradation pathway of the lysosome, is associated with many physiological and pathological processes. The hallmark of autophagy is the formation of the autophagosome that engulfs and degrades cytosolic components via its fusion with the lysosome, in either a selective or a non-selective manner. Autophagy is tightly regulated by proteins encoded by autophagy-related (atg) genes. Among these proteins, ATG8/ LC3 is essential for autophagosome biogenesis/maturation and it also functions as an adaptor protein for selective autophagy. In mammalian cells, several homologs of yeast Atg8 such as MAP1LC3, GABARAP, and GABARAPL $1 / 2$ have been identified. However, the biological relevance of this gene diversity in higher eukaryotes, and their specific roles, are largely unknown. In this review, we describe the mammalian ATG8/LC3 family and discuss recent advancements in understanding their roles in the autophagic process. [BMB Reports 2016; 49(8): 424-430]
\end{abstract}

\section{INTRODUCTION}

Autophagy is an evolutionarily conserved, highly regulated lysosomal degradative pathway for cellular components, which is essential for cellular maintenance and cell viability (1). Autophagy functions to eliminate unnecessary macromolecules, damaged organelles, and intracellular pathogens through the fusion of the lysosome with a double-membrane bound autophagosome, which can also sequester cargo (2). Following degradation, breakdown products are recycled in order to provide a source of metabolites that can be re-used to protect vital cell structure and functions under conditions of stress. This

${ }^{*}$ Corresponding author. Tel: +82-42-629-8785; Fax: +82-42-6298769; E-mail: leeja@hnu.kr

http://dx.doi.org/10.5483/BMBRep.2016.49.8.081

Received 10 May 2016, Revised 3 June 2016, Accepted 29 June 2016

Keywords: ATG8, Autophagy, GABARAP, GABARAPL, LC3 process is also involved in many physiological processes within cells, such as cellular differentiation, development, maintenance, and immunity, and its dysregulation is linked to many different human diseases including cancer, infectious diseases, heart diseases, liver diseases, and several neurological disorders (3). Although autophagy is generally considered as a non-selective degradative pathway, a growing body of evidence suggests that selective autophagy, with cargo specificity, also exists. Examples of the latter include mitophagy, pexophagy, aggrephagy, and xenophagy (4). Besides the degradative role of autophagy, more recently, an autophagy-based secretory pathway has been reported thus expanding its functions to non-degradative pathways (5).

Upon autophagy induction by various cellular stresses such as nutrient-deprivation, or accumulation of misfolded proteins, a cup-shaped double membrane structure referred to as isolation membrane is formed from intracellular membrane sources employing multiple autophagy-related (Atg) proteins to the membrane formation site. After elongation and sealing of the autophagosome, it matures into either an amphisome or an autolysosome through fusion with the endosome or lysosome, respectively. In the autolysosome, the cytosolic cargo is finally degraded by several lysosomal hydrolases. During the autophagic process, autophagosome formation and maturation is regulated by several Atg core proteins in a highly controlled manner (6). Among several Atg core proteins, the Atg8/ MAP1LC3 (microtubule associated protein 1 light-chain 3, hereafter referred to as LC3) conjugation system is required for elongation and maturation of the autophagosome. Atg8/LC3 is cleaved by ATG4(B) protease to expose a C-terminal glycine residue. Cleaved LC3 is then conjugated to phosphatidylethanolamine (PE) by sequential activation of Atg7 (E1-like enzyme), Atg3 (E2-like enzyme), and the Atg12 complex, to generate LC3-PE (a membrane-bound form of LC3 also referred to as LC-II), the level of which is known to be correlated with the number of autophagosomes (Figs. 1, 2). The cellular localization of Atg8/LC3 in the autophagosome has been associated with closure, hemi-fusion, or transport of the autophagosome during its maturation. LC3 can itself be degraded by autophagy indicating that it is an autophagic substrate. Henceforth, LC3 has

ISSN: 1976-670X (electronic edition)

Copyright (c) 2016 by the The Korean Society for Biochemistry and Molecular Biology

(c) This is an open-access article distributed under the terms of the Creative Commons Attribution Non-Commercial License (http://creativecommons.org/licenses/by-nc/4.0) which permits unrestricted non-commercial use, distribution, and reproduction in any medium, provided the original work is properly cited. 


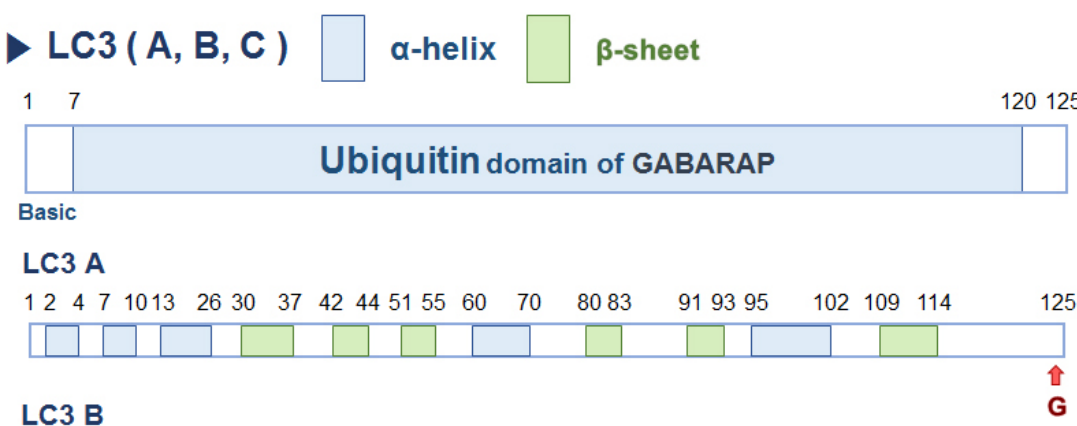

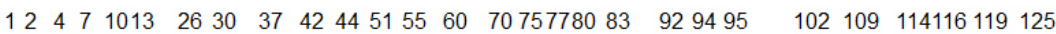

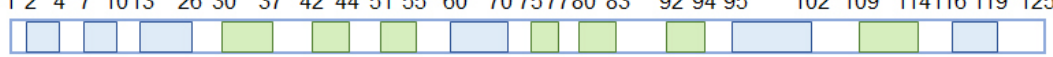

\section{LC3 C}

$1 \quad 131619 \quad 32 \quad 36 \quad 43 \quad 4850 \quad 56 \quad 6166 \quad 76777981838689$

10110811111

\section{G}

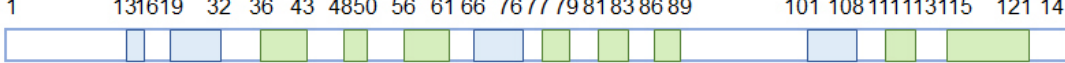

菅

\section{GABARAP}

\begin{tabular}{|l|l|l|}
\multicolumn{2}{c}{22} & 36 \\
\hline $\begin{array}{c}\text { Interaction } \\
\text { with } \beta \text {-tubulin }\end{array}$ & $\begin{array}{l}\text { Interaction } \\
\text { with GPHN }\end{array}$ & Interaction with GABRG2 \\
\hline
\end{tabular}

Acidic

\section{GABARAP}

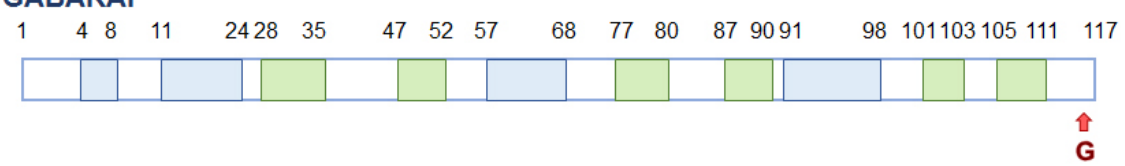

\section{GABARAPL1}

$22 \quad 36$

68

Interaction Interaction

with $\beta$-tubulin with GABRG2

Neutral

\section{GABARAPL1}

\begin{tabular}{|l|l|l|l|l|l|l|l|l|l|l|l|l|l|l|l|l|l|l|l|l|}
1 & 4 & 8 & 11 & 24 & 28 & 35 & 48 & 52 & 57 & 67 & 77 & 80 & 91 & 98 & 105 & 110 & 117 \\
\hline & & & & & & & & & & & & & & & & & & \\
\hline
\end{tabular}

GABARAPL2

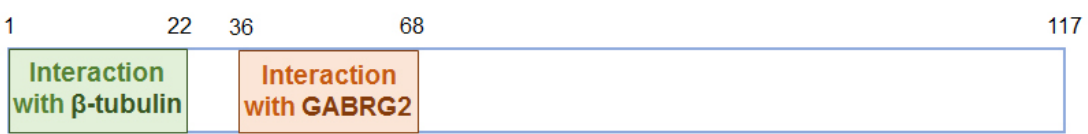

\section{Neutral}

\section{GABARAPL2}

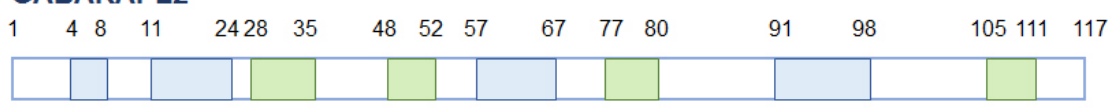

Fig. 1. Domain structure of mammalian ATG8/LC3 family proteins. ATG8/LC3 family has an amino-terminal helix and a C-terminal ubiquitin core composed of $\beta$-strands with hydrophobic pockets. LC3/GABARAP(L)-I cleaved by ATG4 protease is conjugated to phosphatidylethanolamine (PE) to generate LC3II-PE. The arrow indicates the cleavage site of LC3/GABARAP(L) family proteins. 

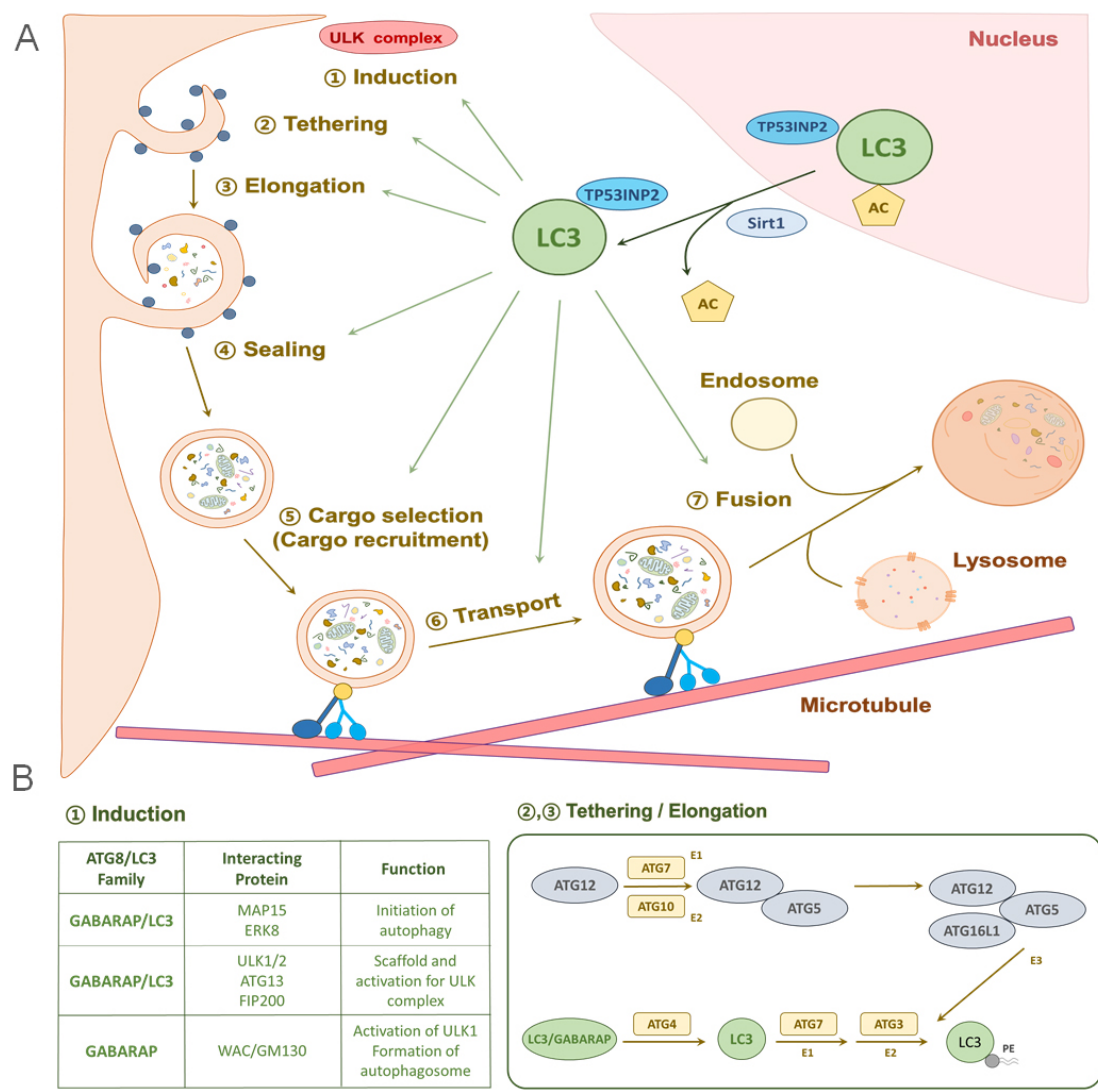

(5) Cargo selection (Cargo recruitment)

\begin{tabular}{|c|c|c|}
\hline $\begin{array}{l}\text { ATG8/LC3 } \\
\text { Family }\end{array}$ & $\begin{array}{l}\text { Interacting } \\
\text { Protein }\end{array}$ & Function \\
\hline LC3C & NDP52 & $\begin{array}{c}\text { Receptor for } \\
\text { ubiquitinated bacteria }\end{array}$ \\
\hline \multirow{3}{*}{ GABARAP } & Multan & Regulation of mitophagy \\
\hline & NBR1 & $\begin{array}{l}\text { Cargo receptor for misfolded } \\
\text { proteins / aggregates }\end{array}$ \\
\hline & ALFY & $\begin{array}{l}\text { Recruitment of ALFY into } \\
\text { LC3B containing structure }\end{array}$ \\
\hline GABARAPL1 & BNIP3L & $\begin{array}{l}\text { Adaptor for autophagy } \\
\text { receptor (mitophagy) }\end{array}$ \\
\hline $\begin{array}{c}\text { LC3B/ } \\
\text { GABARAPL1/2 }\end{array}$ & Dishevelled2 & Negative regulation of Wnt \\
\hline
\end{tabular}

(7) Fusion

\begin{tabular}{|c|c|c|}
\hline $\begin{array}{c}\text { ATG8/LC3 } \\
\text { Family }\end{array}$ & $\begin{array}{c}\text { Interacting } \\
\text { Protein }\end{array}$ & Function \\
\hline GABARAP/LC3 & $\begin{array}{c}\text { Rab7 } \\
\text { FYCO1 }\end{array}$ & $\begin{array}{c}\text { Fusion of autophagosome } \\
\text { with lysosome }\end{array}$ \\
\cline { 2 - 3 } & $\begin{array}{c}\text { TBC1D } \\
\text { VPS29 }\end{array}$ & $\begin{array}{c}\text { Endosome fusion } \\
\text { vesicular transport }\end{array}$ \\
\hline GABARAP & PLEKHM1 & $\begin{array}{c}\text { Fusion of autophagosome } \\
\text { via HOPS }\end{array}$ \\
\hline GABARAP & PI4K2A/PI4KIII $\alpha$ & $\begin{array}{c}\text { Targeting of } \\
\text { PI4K2A/PI4LIIl into } \\
\text { autophagosome }\end{array}$ \\
\hline
\end{tabular}

Fig. 2. Differential/compensatory roles of the LC3 and GABARAP subfamilies during autophagy. (A) Autophagy can be induced in response to a variety of cellular stresses. Once autophagy is activated, it is executed in a multi-step processes including induction, formation elongation/sealing of the isolation membrane, cargo selection/recruitment, transport/ fusion of the autophagosome, and cargo degradation in the autolysosome. (B) LC3, GABARAP, or GABARAPL, together with the autophagy core machinery proteins or specific proteins, regulate autophagosome biogenesis, cargo recognition/recruitment, transport, and fusion. Some functions, such as elongation of the membrane or cargo recruitment, are redundant between these proteins; however, other functions such as fusion or recruitment of specific cargo are differentially regulated by each ATG8/LC3 protein together with its interacting partners. been widely used to monitor the number of autophagosomes as well as autophagic activity. Moreover, emerging evidence has shown that during selective autophagy, LC3 functions as an adaptor protein to recruit selective cargo to the autophagosome via interaction with cargo receptors (7). LC3 also regulates a process referred to as LAP (LC3-associated phagocytosis), which does not have the classic double membrane autophagosome. Instead, a single-membrane structure is generated, which is involved in the efficient clearance of dead cells (8). Although there is only one Atg8 protein in yeast, there are several mammalian Atg8 orthologs, which are classified into the LC3, GABARAP ( $\gamma$-aminobutyric acid receptorassociated protein), and GABARAPL ( $\gamma$-aminobutyric acid receptor-associated protein like) subgroups (Fig. 1) (9). Since the discovery of mammalian homologs of Atg8, functional characterization has mostly been focused on LC3. The exact functions of each of the mammalian Atg8 paralogs and their biological roles in autophagy in various cellular contexts are largely unknown and many key questions remain unanswered. For example; Is there any spatiotemporal specificity in paralog expression? Where are the different paralogs localized? How are the paralogs involved in different types of autophagy? Therefore, an understanding of the role of paralogs in autophagy as well autophagy-associated pathways will provide a more sophisticated understanding of the regulation of autophagic processes. Such knowledge may lead to the 
identification of new molecular targets that are important in modulating autophagy in several human diseases. In this review, based on recent advancements in studies involving Atg8/LC3 family, we will focus on the structures and the physiological roles of the mammalian Atg8/LC3 family in autophagy, and finally discuss their potential involvement in the cellular pathogenesis of human diseases.

\section{ATG8/LC3 family: expression \& structure}

Yeast Atg8, is an ubiquitin-like (Ubl) protein, exhibiting amino acid sequence similarity with LC3 (A, B, C), GABARAP, GABARAPL1(GEC1), GABARAPL2 (also known as GATE-16 (Golgi-associated ATPase enhancer of $16 \mathrm{kDa})$ ), and GABARAPL3 (Fig. 1) (10). The number of genes has probably arisen owing to gene duplication and loss events during evolution (9).

Among the mammalian homologs of Atg8, LC3 was first identified as the light chain of microtubule-associated protein $1 \mathrm{~A}$ and $1 \mathrm{~B}$ in the rat brain (LC3A and LC3B); however, its exact function in cellular transport was considered necessary to be clarified. Since then, the role of LC3 in autophagy was first described by Yoshimori's group and it has been well characterized as compared to other LC3 paralogs (11). In addition, LC3C has been recently reported to have a role in autophagosome formation and COPII (coat protein complex II)-dependent ER export via binding to TECPR2 (tectonin $\beta$-propeller containing protein 2), which could possibly represent a link between the autophagic and the secretory pathways (12).

GABARAP and GABARAPL were initially characterized as intracellular trafficking factors (9). GABARAP has been shown to interact with a myriad of binding partners, including the gamma2 subunit of the GABAA receptor, tubulin and microtubules, the $\mathrm{N}$-ethyl maleimide sensitive factor, gephyrin, and the transferrin receptor (13). GABARAP has been characterized in the context of being a $G A B A(A)$ receptorassociated protein and in particular, it has been extensively studied in the regulation of neuronal signaling receptors (14). Recently, GABARAP has also been suggested to be a membrane-localized signaling scaffold that regulates TIAM1 (T lymphoma invasion and metastasis 1)-RAC1 signaling via association with the CUL3 (Culin3)-KBTBD6/7 (Kelch repeat and BTB domain-containing protein 6/7) ubiquitin ligase (15). GABARAPL1 (also known as GEC1 (glandular epithelial cell 1)) was first identified as an early estrogen-induced gene in quiescent guinea-pig endometrial GECs (16). Interestingly, GABARAPL1 has been implicated in several cellular processes involved in the specific regulation of estrogen hormones, the forkhead box $\mathrm{O}$ (FOXO) family, and circadian rhythm. A dysregulation of GABARAPL1 expression is associated with breast cancer, colorectal cancer, and neurodegenerative disease such as Parkinson's diseases (17). GABARAPL2 regulates intra-Golgi transport by linking NSF (N-ethylmaleimide-sensitive factor) and SNAREs (Soluble NSF Attachment Protein Receptors) and plays a role in post-mitotic Golgi reassembly
(18).

Although Atg8/LC3 family members are ubiquitously expressed, there is some tissue specificity in their expression. The mRNAs for GABARAPL1 or GABARAPL2 are predominantly expressed in the central nervous system especially the brain. Interestingly, GABARAPL1 is expressed specifically in the regions important for regulating somatomotor or endocrine function, such as the pons or diencephalon, suggesting a crucial role in these processes (19). In contrast, the expression of GABARAP is found to be much higher in endocrine glands. Interestingly, among the ATG8/LC3 family members, LC3C expression is found to be relatively low in nearly all tissues examined with the exception of lung, where it is highly expressed (18). The expression of individual ATG8/LC3 protein has been proposed to be regulated at the transcriptional, post-transcriptional, or post-translational level (20).

All Atg8/LC3 proteins have an ubiquitin-like structure despite differences in their respective amino-acid sequences. The amino-terminus is composed of an $\alpha$-helix, which differs among the Atg8/LC3 members, whereas the conserved carboxy-terminus consists of an ubiquitin core composed of $\beta$-strands with hydrophobic pockets (Fig. 1) (7). The C-terminal conserved ubiquitin core region is responsible for the interaction of protein with Atg core machinery proteins, such as the conjugation system. Therefore, it is hypothesized that the divergent $\mathrm{N}$-terminal $\alpha$-helices might be important for specific functions during autophagy via protein-protein interactions, lipid-protein interactions, or through post-translational modifications (Fig. 1). Consistent with this concept, it has been reported that the first $\alpha$-helix in LC3 is basic in nature, whereas the same in GABALAPL2 or GABARAP is neutral or acidic, respectively (21). As is the case for LC3-I and LC3-II, all Atg8/LC3 proteins exist in two forms, namely cytosolic or membrane bound, suggesting that the processing of ATG8/LC3 family members is conserved (Fig. 2). ATG8/LC3 proteins are processed by Atg4 proteins and consistent with the presence of mammalian ATG8/LC3 paralogs, there are multiple homologs of Atg4. Atg4A is specific for GABARAP and GABARAPL2, whereas Atg4B appears to be involved in processing of all ATG8/LC3 family members although with different affinities.

Several ATG8/LC3 protein-interacting molecules have been identified and characterized in selective or nonselective autophagy. Many ATG8/LC3 interacting proteins have a basic hydrophobic AIM/LIR (ATG8-interacting motif/LC3-interacting region) motif with a core sequence of (W/F/Y)XX(L/I/V) (22). The functions of ATG8/LC3 proteins appear to depend on binding to effector proteins in a LIR-dependent manner. In the following section, we will describe the functional role of each ATG8/LC3 protein during autophagy.

\section{Physiological functions of the ATG8/LC3 family in autophagy Autophagosome biogenesis}

Initial step: LC3 is localized to both the nucleus and the cytosol implying that the protein can shuttle between the two 
cellular compartments. Recent studies have shown that during starvation, deacetylation of LC3 by Sirt1 (Sirtuin 1) and association of LC3 with the nuclear tumor protein TP53INP2 (p53 inducible nuclear protein 2) promotes LC3 redistribution from the nucleus to the cytosol, where it associates with Atg7 and the autophagy core machinery to form autophagosomes (Fig. 2) (23).

LC3 and GABARAP proteins are associated with the serine-threonine kinases ULK1/2 (uncoordinated-51-like kinase 1/2), Atg13, and FIP200 (FAK family kinase-interacting protein of $200 \mathrm{kDa}$ ), and are contemplated to act as scaffolds for the assembly of the ULK complex (24). Interestingly, GABARAP displays a binding preference for the ULK complex when compared to the LC3 subfamily (24). More recently it has been reported that, during starvation-induced autophagy, trafficking of GABARAP from the centrosome (but not LC3, GABARAPL1, or GABARAP2) specifically promotes ULK activation, which subsequently mediates non-hierarchical function in the formation of the autophagosome via regulation of WAC (WW domain-containing adaptor with coiled coil) and GM130 (Golgi matrix protein of $130 \mathrm{kDa}$ ) proteins (25). Moreover, MAPK15/ERK8 stimulates autophagy by interacting with LC3 and GABARAP proteins, indicating that MAPK15/ERK8 is an upstream regulator of autophagosome formation that functions by recruiting LC3 and GABARAP to the autophagosome formation site (Fig. 2B) (26).

Late step: Based on studies employing yeast Atg8, it is evident that ATG8/LC3 can promote membrane fusion, elongation, and sealing of the autophagosome during autophagosome biogenesis (Fig. 2B) (27). Expression of a kinase-dead mutant of ATG4B in mammalian cells led to accumulation of unclosed pre-autophagosomal membrane structures, suggesting that mammalian homologs contribute to the closure of the isolation membrane and the formation of autophagosome (28). However, experiments wherein each ATG8/LC3 family member was knocked down using specific siRNAs have indicated that each ATG8/LC3 protein regulates a different step of autophagosome formation. LC3 mediates the elongation step of the isolation membrane, whereas GABARAP and GABARAPL2 are involved in the later steps of autophagosome formation, such as sealing or fusion (29). More recently, it has been reported that GABARAP-mediated targeting of PI4K2A (phosphatidylinositol 4-kinase type 2 alpha, also abbreviated as PI4KII $\alpha$ ) to autophagosomes regulates Ptdlns4P-dependent autophagosomelysosome fusion, thus supporting the role of GABARAP in the maturation of autophagosomes (Fig. 2B) (30). Furthermore, LC3/GABARAP proteins have been reported to be associated with PLEKHM1 (Pleckstrin homology domain containing protein family member 1 ) and to regulate autophagosomelysosome fusion through the HOPS (homotypic fusion and vacuole protein sorting) complex (Fig. 2B) (31). Interestingly, based on a recent study using a minimally reconstituted system, both enzymatically and chemically lipidated forms of GABARAPL2 and GABARAP proteins were shown to promote extensive membrane tethering and fusion, whereas lipidated forms of LC3 performed similar function to a much lesser extent. This finding suggests a preferential role for the GABARAP subfamily in membrane fusion as compared to LC3 subfamily (15). Moreover, LC3 indirectly regulates intracellular trafficking of the autophagosome for its fusion with the lysosome. Rab7 and its interacting protein, FYCO1 (FYVE and coiled-coil domain containing protein 1) are known to regulate the transport of autophagosomes to lysosomes by interacting with LC3 and kinesin motor proteins (Fig. 2B) (32).

Intriguingly, several members of TBC (Tre-2/Bub2/Cdc16), a family of GAPs (GTPase-activating proteins), interact with LC3/GABARAP proteins suggesting their important roles during autophagy and vesicular trafficking. Indeed, TBC1D5 (TBC1 domain family, member 5), which has two- LIR motifs, has been reported to associate with VPS 29 (vacuolar protein sorting protein 29), a retromer complex protein, thereby establishing linkage between autophagy and intracellular trafficking pathways (Fig. 2B) (33).

\section{Cargo recognition/recruitment during selective autophagy of the ATG8/LC3 family}

ATG8/LC3 family also has an extensive role in cargo recognition and selective targeting to autophagosomes through specific interactions with autophagy receptors during selective autophagy $(22,34)$. Autophagy receptors have a LIR motif, which mediates association with the Ubl domain of ATG8/LC3 proteins. This motif usually recognizes ubiquitinated cargo such as protein aggregates, damaged mitochondria, or peroxisomes, and targets them to autophagosomes. Since the first discovery of p62, a multifunctional protein that is an autophagy receptor for ubiquitinated protein aggregates and many ATG8/LC3 proteins have been identified as selective autophagy receptors. Selective autophagy receptors include NBR1 (neighbor of BRCA1) for protein aggregates, NDP52 (nuclear dot protein of $52 \mathrm{kDa}$ ) and optineurin for intracellular pathogens, and Nix, BNIP3 (BCL2/adenovirus E1B 19 kDa interacting protein), BNIP3L, and FUNDC1 (Fun14 domain containing 1) for recognizing outer mitochondrial membrane proteins for the recruitment of the autophagic machinery to damaged mitochondria $(9,35)$. Moreover, certain extent of specificity exists in the association between ATG8/LC3 proteins and the selective autophagy receptors, although the mechanism underlying specificity remains to be understood. For example, NDP52 shows preferential binding to LC3C, rather than GABARAP, or GABARAPL; whereas, BNIP3L preferentially associates with GABARAPL1, rather than LC3. Binding of GABARAP to ALFY (autophagy-linked FYVE protein) is required for selective binding and the recruitment of ALFY to LC3B-positive structures (36). GABARAP regulates mitophagy via its association with the E3 ubiquitin ligase Mulan, which can interact with multiple E2-conjugating enzymes (37). LC3B/GABARAP/GABARAPL1 associate with Dvl2 (Dishevelled2), which negatively regulates the $\mathrm{Wnt}$ 
pathway (38). How specific interactions between each ATG8/LC3 protein and selective autophagy receptors are regulated, and how these interactions cooperate in a contextdependent manner will be an interesting future area of study.

\section{REGULATION OF ATG8/LC3 PROTEINS}

ATG8/LC3, like other Atg core proteins can be tightly regulated at the transcriptional, post-transcriptional, or posttranslational level. Recently, various transcription factors such as ATF4 (activating transcription factor 4), CEBP $\beta$ (CCAATenhancer-binding protein $\beta$ ), CHOP (C/EBP homologous protein), E2F1 (E2F Transcription Factor 1), FOXO 1/3, GATA1 (GATA Binding Protein 1 or Globin Transcription Factor 1), Jun, TFEB (transcription factor EB, SREBF2/SREBP2 (sterol regulatory element-binding protein 2), and ZKSCAN3 (Zinc finger protein with KRAB and SCAN domains 3) have been reported as transcriptional regulators of the ATG8/LC3 family as well as other Atg genes (20). Interestingly, among these transcription factors, only GATA1 (which activates genes involved in lysosome biogenesis and function) has been reported to regulate the ATG8/LC3 family during hematopoiesis (39). In addition, microRNAs such as MiR-204 have been reported to be involved in LC3B-mediated autophagy at the post-transcriptional level in renal clear cell carcinoma (40).

Among post-translational modifications, phosphorylation and acetylation of ATG8/LC3 family members have been reported. The phosphorylation of the $\mathrm{N}$-terminus of LC3B by PKA is known to be negatively regulated upon autophagy induction (41). More recently, it has been reported that the hippo kinase STK3 (Serine/threonine kinase 3) phosphorylates LC3 and regulates autophagosome fusion with the lysosome (42). Acetylation and deacetylation of LC3 by EP300 (E1A binding protein 300/p300) can also modulate autophagic activity. Deacetylation of LC3 promotes autophagy, whereas acetylation inhibits the autophagic process (43). Other post-translational modifications such as ubiquitination and SUMOylation of other essential autophagy proteins have also been extensively characterized. Further studies on the spatiotemporal regulation of each ATG8/LC3 at the transcriptional, post-transcriptional, and post-translational level are required to gain in-depth understanding on the role of mammalian autophagy.

\section{CONCLUSIONS AND PERSPECTIVES}

Each mammalian ATG8/LC3 protein acts as a scaffold for autophagy core machinery and functions in autophagosome biogenesis, as an adaptor of selective cargo for recognition/ recruitment to the autophagosome, or as a regulator for the activity of signaling proteins such as kinase/GTPase/GAP, or as a decision maker for cell fate.

However, many key questions still remain unanswered. Certain issues which need to be clarified are as follows: How is the differential interaction between mammalian homologs of Atg8 and other Atg core proteins or other cellular proteins regulated? What determines the specificity of their functions? What is the biological relevance of these interactions in vivo? Do defects in mammalian ATG8/LC3 proteins contribute to pathogenic effects during disease progression in many autophagy-associated human diseases? Recent accumulating evidence demonstrates alteration of level or distribution of LC3 and GABARAP in neurodegenerative diseases such as Lewy body diseases as well as in some cancers (44). Therefore, in future, elucidation of alterations in specific ATG8/LC3 forms in human diseases could provide insights for the discovery, diagnosis, or targeting of several autophagy-related human diseases.

\section{ACKNOWLEDGEMENTS}

We apologize to colleagues whose work could not be cited due to space limitations. This work was supported by the National Honor Scientist Program, National Research Foundation (2014R1A1A4A01003859), and Korea Health Technology R\&D project (HI14C1891) to J.-A L.

\section{REFERENCES}

1. Klionsky DJ and Codogno P (2013) The mechanism and physiological function of macroautophagy. J Innate Immun 5, 427-433

2. Klionsky DJ (2007) Autophagy: from phenomenology to molecular understanding in less than a decade. Nat Rev Mol Cell Biol 8, 931-937

3. Schneider JL and Cuervo AM (2014) Autophagy and human disease: emerging themes. Curr Opin Genet Dev 26, 16-23

4. Ktistakis NT and Tooze SA (2016) Digesting the Expanding Mechanisms of Autophagy. Trends Cell Biol 26, 624-635

5. Ponpuak M, Mandell MA, Kimura T, Chauhan S, Cleyrat C and Deretic V (2015) Secretory autophagy. Curr Opin Cell Biol 35, 106-116

6. Xie Z and Klionsky DJ (2007) Autophagosome formation: core machinery and adaptations. Nat Cell Biol 9, 1102-1109

7. Rogov V, Dotsch V, Johansen T and Kirkin V (2014) Interactions between autophagy receptors and ubiquitinlike proteins form the molecular basis for selective autophagy. Mol Cell 53, 167-178

8. Martinez J, Almendinger J, Oberst A et al (2011) Microtubule-associated protein 1 light chain 3 alpha (LC3)-associated phagocytosis is required for the efficient clearance of dead cells. Proc Natl Acad Sci U S A 108, 17396-17401

9. Slobodkin MR and Elazar Z (2013) The Atg8 family: multifunctional ubiquitin-like key regulators of autophagy. Essays Biochem 55, 51-64

10. Xin Y, Yu L, Chen Z et al (2001) Cloning, expression 
patterns, and chromosome localization of three human and two mouse homologues of GABA(A) receptorassociated protein. Genomics 74, 408-413

11. Kabeya $\mathrm{Y}$, Mizushima N, Yamamoto A, OshitaniOkamoto S, Ohsumi Y and Yoshimori T (2004) LC3, GABARAP and GATE16 localize to autophagosomal membrane depending on form-II formation. J Cell Sci 117, 2805-2812

12. Stadel D, Millarte V, Tillmann KD et al (2015) TECPR2 Cooperates with LC3C to Regulate COPII-Dependent ER Export. Mol Cell 60, 89-104

13. Coyle JE and Nikolov DB (2003) GABARAP: lessons for synaptogenesis. Neuroscientist 9, 205-216

14. Chen ZW and Olsen RW (2007) GABAA receptor associated proteins: a key factor regulating GABAA receptor function. J Neurochem 100, 279-294

15. Engedal N and Seglen PO (2016) Autophagy of cytoplasmic bulk cargo does not require LC3. Autophagy $12,439-441$

16. Pellerin I, Vuillermoz $C$, Jouvenot $M$, Ordener $C$, Royez $M$ and Adessi GL (1993) Identification and characterization of an early estrogen-regulated RNA in cultured guinea-pig endometrial cells. Mol Cell Endocrinol 90, R17-21

17. Le Grand JN, Chakrama FZ, Seguin-Py S et al (2011) GABARAPL1 (GEC1): original or copycat? Autophagy 7, 1098-1107

18. Sagiv Y, Legesse-Miller A, Porat A and Elazar Z (2000) GATE-16, a membrane transport modulator, interacts with NSF and the Golgi v-SNARE GOS-28. EMBO J 19, 1494-1504

19. Tolle F, Risold PY, Mansuy-Schlick V et al (2008) Specific regional distribution of gec1 mRNAs in adult rat central nervous system. Brain Res 1210, 103-115

20. Feng Y, Yao Z and Klionsky DJ (2015) How to control self-digestion: transcriptional, post-transcriptional, and post-translational regulation of autophagy. Trends Cell Biol 25, 354-363

21. Sugawara K, Suzuki NN, Fujioka Y, Mizushima N, Ohsumi $Y$ and Inagaki $F$ (2004) The crystal structure of microtubule-associated protein light chain 3, a mammalian homologue of Saccharomyces cerevisiae Atg8. Genes Cells 9, 611-618

22. Birgisdottir $A B$, Lamark $T$ and Johansen $T$ (2013) The LIR motif - crucial for selective autophagy. J Cell Sci 126 , 3237-3247

23. Huang R, Xu Y, Wan W et al (2015) Deacetylation of nuclear LC3 drives autophagy initiation under starvation. Mol Cell 57, 456-466

24. Alemu EA, Lamark T, Torgersen KM et al (2012) ATG8 family proteins act as scaffolds for assembly of the ULK complex: sequence requirements for LC3-interacting region (LIR) motifs. J Biol Chem 287, 39275-39290

25. Joachim J, Jefferies HB, Razi $M$ et al (2015) Activation of ULK Kinase and Autophagy by GABARAP Trafficking from the Centrosome Is Regulated by WAC and GM130. Mol Cell 60, 899-913

26. Colecchia D, Strambi A, Sanzone S et al (2012) MAPK15/ERK8 stimulates autophagy by interacting with LC3 and GABARAP proteins. Autophagy 8, 1724-1740
27. Feng Y, He D, Yao Z and Klionsky DJ (2014) The machinery of macroautophagy. Cell Res 24, 24-41

28. Fujita N, Hayashi-Nishino M, Fukumoto $\mathrm{H}$ et al (2008) An Atg4B mutant hampers the lipidation of LC3 paralogues and causes defects in autophagosome closure. Mol Biol Cell 19, 4651-4659

29. Weidberg $H$, Shvets E, Shpilka T, Shimron F, Shinder $V$ and Elazar Z (2010) LC3 and GATE-16/GABARAP subfamilies are both essential yet act differently in autophagosome biogenesis. EMBO I 29, 1792-1802

30. Albanesi J, Wang $\mathrm{H}$, Sun HQ, Levine B and Yin $\mathrm{H}$ (2015) GABARAP-mediated targeting of PI4K2A/PI4KIlalpha to autophagosomes regulates Ptdlns4P-dependent autophagosome-lysosome fusion. Autophagy 11, 2127-2129

31. McEwan DG, Popovic D, Gubas A et al (2015) PLEKHM1 regulates autophagosome-lysosome fusion through HOPS complex and LC3/GABARAP proteins. Mol Cell 57, 39-54

32. Pankiv S, Alemu EA, Brech A et al (2010) FYCO1 is a Rab7 effector that binds to LC3 and PI3P to mediate microtubule plus end-directed vesicle transport. J Cell Biol 188, 253-269

33. Popovic D, Akutsu M, Novak I, Harper JW, Behrends C and Dikic I (2012) Rab GTPase-activating proteins in autophagy: regulation of endocytic and autophagy pathways by direct binding to human ATG8 modifiers. Mol Cell Biol 32, 1733-1744

34. Wild P, McEwan DG and Dikic I (2014) The LC3 interactome at a glance. J Cell Sci 127, 3-9

35. Kalvari I, Tsompanis S, Mulakkal NC et al (2014) iLIR: A web resource for prediction of Atg8-family interacting proteins. Autophagy 10, 913-925

36. Lystad AH, Ichimura Y, Takagi K et al (2014) Structural determinants in GABARAP required for the selective binding and recruitment of ALFY to LC3B-positive structures. EMBO Rep 15, 557-565

37. Ambivero CT, Cilenti L, Main S and Zervos AS (2014) Mulan E3 ubiquitin ligase interacts with multiple E2 conjugating enzymes and participates in mitophagy by recruiting GABARAP. Cell Signal 26, 2921-2929

38. Gao C, Cao W, Bao L et al (2010) Autophagy negatively regulates Wnt signalling by promoting Dishevelled degradation. Nat Cell Biol 12, 781-790

39. Kang YA, Sanalkumar R, O'Geen H et al (2012) Autophagy driven by a master regulator of hematopoiesis. Mol Cell Biol 32, 226-239

40. Xiao J, Zhu X, He B et al (2011) MiR-204 regulates cardiomyocyte autophagy induced by ischemia-reperfusion through LC3-II. J Biomed Sci 18, 35

41. Cherra SJ 3rd, Kulich SM, Uechi G et al (2010) Regulation of the autophagy protein LC3 by phosphorylation. J Cell Biol 190, 533-539

42. Wilkinson DS, Jariwala JS, Anderson E et al (2015) Phosphorylation of LC3 by the Hippo kinases STK3/STK4 is essential for autophagy. Mol Cell 57, 55-68

43. Lee IH and Finkel T (2009) Regulation of autophagy by the p300 acetyltransferase. J Biol Chem 284, 6322-6328

44. Tanji K, Odagiri S, Maruyama A et al (2013) Alteration of autophagosomal proteins in the brain of multiple system atrophy. Neurobiol Dis 49, 190-198 